\title{
Region Specific Election Routing Protocol for Wireless Sensor Networks
}

\author{
S. Koteeswaran*, R. Srinivasan and K. A. Varunkumar \\ Department of CSE, Vel Tech Rangarajan Dr. Sagunthala R \& D Institute of Science and Technology (Vel Tech Dr.RR \& \\ Dr.SR Technical University), Chennai-62. TamilNadu, India; \\ s.koteeswaran@gmail.com,srinivasanrajkumar28@gmail.com,varun.kumar300@gmail.com
}

\begin{abstract}
Wireless Sensor Networks in real time applications are vastly increasing due to their sensing feature. In this paper we propose an efficient routing protocol for heterogeneous wireless sensor network. Two scenarios are considered: 20 advanced nodes \& 80 normal nodes are used in simulation. In scenario one, Nodes transmitted data directly to the base station. In scenario two, Nodes transmitted their data to base station with assigned cluster node. After assigned rounds are completed, number of alive nodes is considered for analyzing energy efficiency, throughput, stability and network life time. Currently, two efficient routing protocols like Leech, Sep are induced to the simulations and compared with the proposed RSE routing protocol. Results proved that throughput, stability, network life time, energy efficiency are increased in our proposed model.
\end{abstract}

Keywords: Clustering, Energy Efficiency, Election, Regions, Routing, Wireless Sensor Network.

\section{Introduction}

Wireless sensor networks consist of various sensors which are deployed to monitor various activities especially troop movements in battle field and changes in climatic conditions ${ }^{1}$. Now days sensors are used in vehicular ad hoc networks for capturing the speed and distance between next vehicles ${ }^{2}$. Accident sensors are also used to alert the driver when the vehicle is in upside-down stage; sensors are capturing the data and send the information to the master. To send the data to the base station, the network protocol or routing efficient algorithm must be implemented $^{3}$. To ensure the lifetime of node, a protocol chosen for network must be possessed carefully. Sensor nodes lifetime is based on energy dissipation of working node. To maintain the energy, appropriate routing algorithm is needed to be précised and maintained throughout the lifetime of the node 4 . Communication in sensor node is based on the environment which they deployed that prolonged the network scenario and lifetime of the node. The three types of communication techniques used in sensor node are clock driven, event driven, and query driven ${ }^{5}$.

Heterogeneous protocols like Leech which is predominantly used for energy efficient routing protocol which had a same probability for assigning anode to cluster node ${ }^{6}$. A criterion is assumed as round based the node that evolved. The main drawback of Leech is that it does not work well in heterogeneous environment ${ }^{7}$. Lifespan of node is decreased while compare to the other routing protocol' ${ }^{8}$. To overcome this, Stable Election Routing Protocol is introduced; it has different probability for assigning the cluster node among the different nodes. But the energy of the nodes is sustained while comparing to the previous proposed routing protocols?.

\section{Proposed Method}

The proposed method consists of two scenarios: Uninterrupted transmission and transmission through cluster head. Normally nodes are categorized by analyzing 
their energy levels. Region 0, Region 1 and Region 2 are three regions divided according to energy efficiency.

Nodes are categorized three types: normal nodes, advanced nodes, and highly advanced nodes

Region 0 - Normal nodes are deployed in these region because they have less energy while comparing to other nodes

Region 1 - Advanced nodes are deployed.

Region 2 - Highly advanced nodes are deployed.

Reason for deploying advanced nodes and highly advanced nodes in region 1 and region 2 is that the nodes deploying on corners utilized high energy for transmitting data to the base station.

\subsection{RES Operation}

RES Operation is done in two scenarios: - 1) Uninterrupted transmission and 2) Transmission occurs through Cluster head to base station.

In Uninterrupted transmission normal nodes that is least energy efficient nodes transmits their data to base station without any interruption.

\section{Transmission over cluster head:}

Transmission of data to the base station is done by using the clustering algorithms ${ }^{10}$, so for that cluster head has to be selected from the nodes in region 1 and region2. Assigned cluster head collected data from nodes aggregated and sends data to base station. Advanced nodes are deployed in Region 1, Region 2, and Cluster head is formed by the nodes. In equation [1] optimal number of clusters Copt is formed. $\mathrm{N}$ is the number of nodes. According to SEP the probability of cluster node is

$$
x=\frac{\text { Koptimality }}{-n}
$$

Node is assigned with cluster head with certain number of rounds it travels in the Scenario. Whether it is assigned with 0 or 1.If it is lesser than threshold value[n] then the node is does not get the criteria to be a cluster node, or else if it is greater than threshold value it is assigned to cluster node.

$$
T(n)=\frac{\text { Poptimal }}{1-\text { Poptimal }\left(\frac{\text { rxmod } 1}{\text { Poptimal }}\right)} \text { If } \mathrm{N} \varepsilon \mathrm{G}
$$

Where in equation [2] $\mathrm{g}$ is the set of nodes not included in the cluster heads at the probability of the Rounds $\mathrm{N}$

$$
P(\text { advertise })=\frac{\text { Poptimal }}{1+(a, m)}
$$

Equation [3] Cluster head sends the advertisement to the nodes present in the region which has the probability optimality.

$$
\begin{aligned}
& T(a d v)=\frac{\text { Padvertise }}{1-\text { Padvertise }\left(\text { ramod } \frac{1}{\text { Padvertise }}\right)} \\
& \text { If advertise } \varepsilon \mathrm{G}
\end{aligned}
$$

In Equation [4] where $\mathrm{g}$ is defined as set of advanced nodes whether the formation of cluster is done according to the threshold energy level. After the selection of cluster head the nodes have to be known that which clusters head is assigned for particular node. Cluster head broadcasts the advertisement message and it alerts the node whether that particular node has reached to form a network and its belonging node is in active for the command assigned by cluster head ${ }^{11}$.

\section{Simulations}

Simulation of our proposed method is done in MATLAB $2013 \mathrm{Rb}$, with a field dimension of $100 \mathrm{~m} \times 100$ that two regions are separated and 100 nodes are deployed in the two regions, data transmission through the base station, which is situated at center of the network.

Table 1 shows the parameters assigned in the simulation and the values given for the simulation to test the nodes performance. Initial energy, data aggregation energy, energy in advanced nodes, Transmission energy, receiving energy, Probability are the six parameters and different values which are given for the parameters.

Table 1. Simulation Parameters

\begin{tabular}{ll}
\hline Parameters & \multicolumn{1}{c}{ Values } \\
\hline Initial energy & $0.5 \mathrm{j}$ \\
Data aggregation energy & $\mathrm{E}(1+\alpha)$ \\
Energy in advanced nodes & $5 \mathrm{nj} / \mathrm{bit} / \mathrm{signal}$ \\
Transmission energy & $5 \mathrm{nj} / \mathrm{bit}$ \\
Receiving Energy & $5 \mathrm{nj} / \mathrm{bit}$ \\
Probability & 0.1 \\
\hline
\end{tabular}




\section{Results}

Comparison of performance is carried out in the simulation in between Sep and Leech routing protocol. Leech routing protocol have heterogeneity in it's for performing the real time applications ${ }^{12}$. Here in these simulation three routing protocols is compared with same parameters. As a result of these, stability \& heterogeneity of Res is higher than Leech \& Sep protocol.
Throughput of the three routing protocols is examined in the simulation result, from the fig $1, \mathrm{~m}=0.2 \& \alpha=1$. which shows from the 100 nodes assigned for rounds. 20 nodes are advanced nodes \& 80 nodes are normal nodes. In these 20 advanced nodes, 10 nodes are assigned for head region 1 \& another 10 nodes for head region 2.

In simulation, nodes are gone through number of rounds as per this energy efficiency. Figl clearly shows the number of alive nodes after the completion of entire

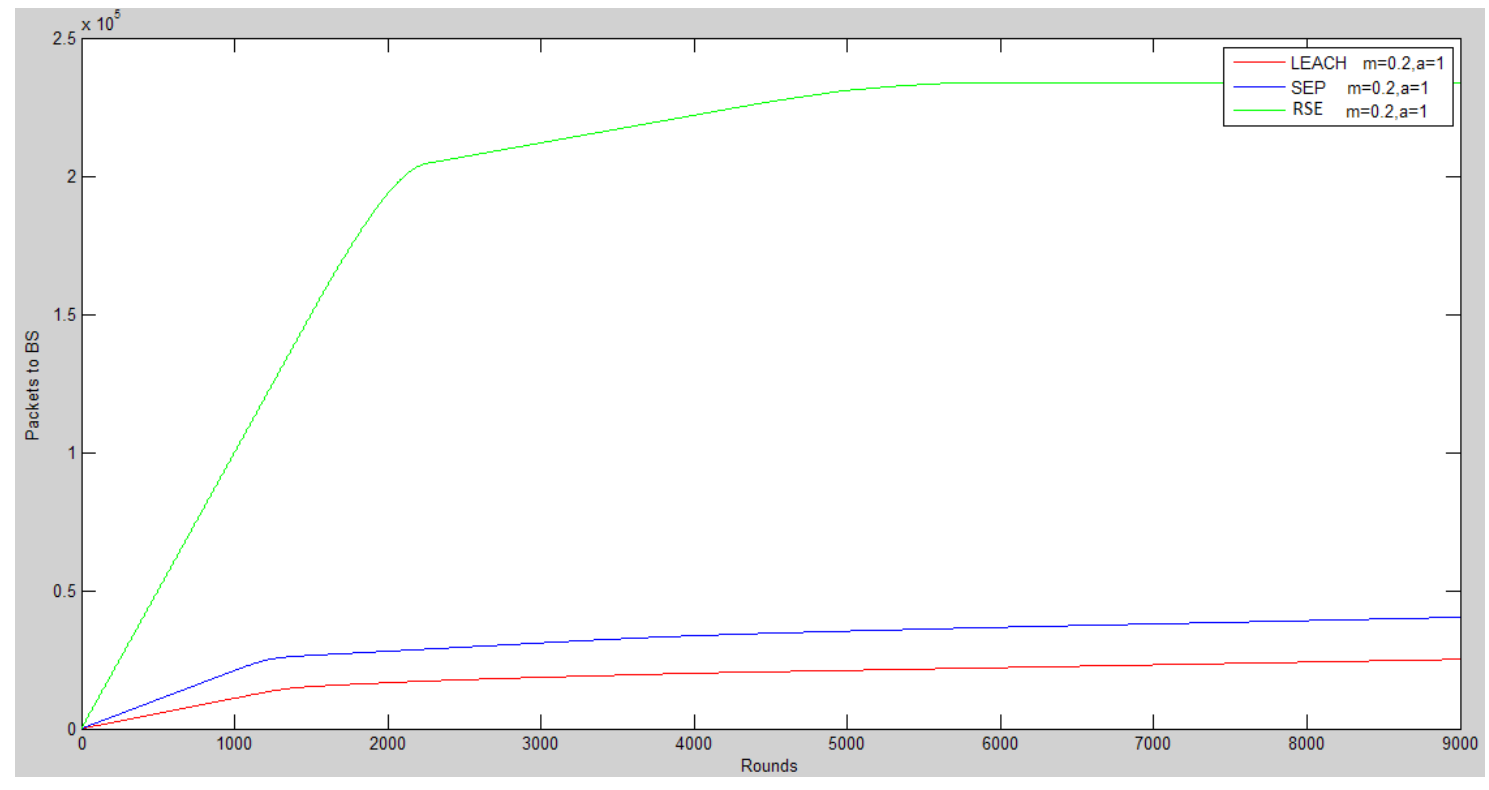

Figure 1. Alive Nodes in LEACH, SEP, RSE.

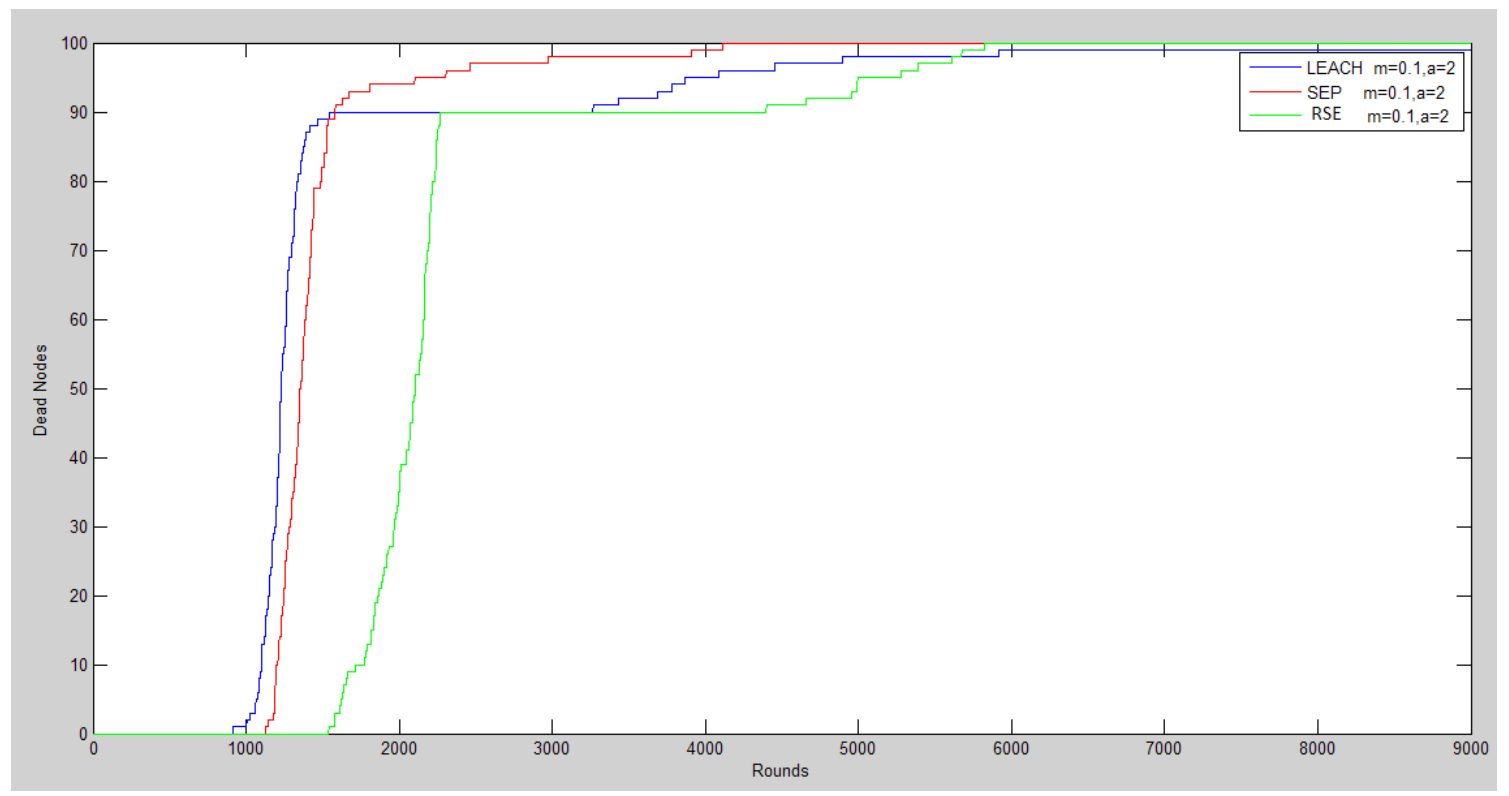

Figure 2. Throughput of LEACH, SEP, RES. 


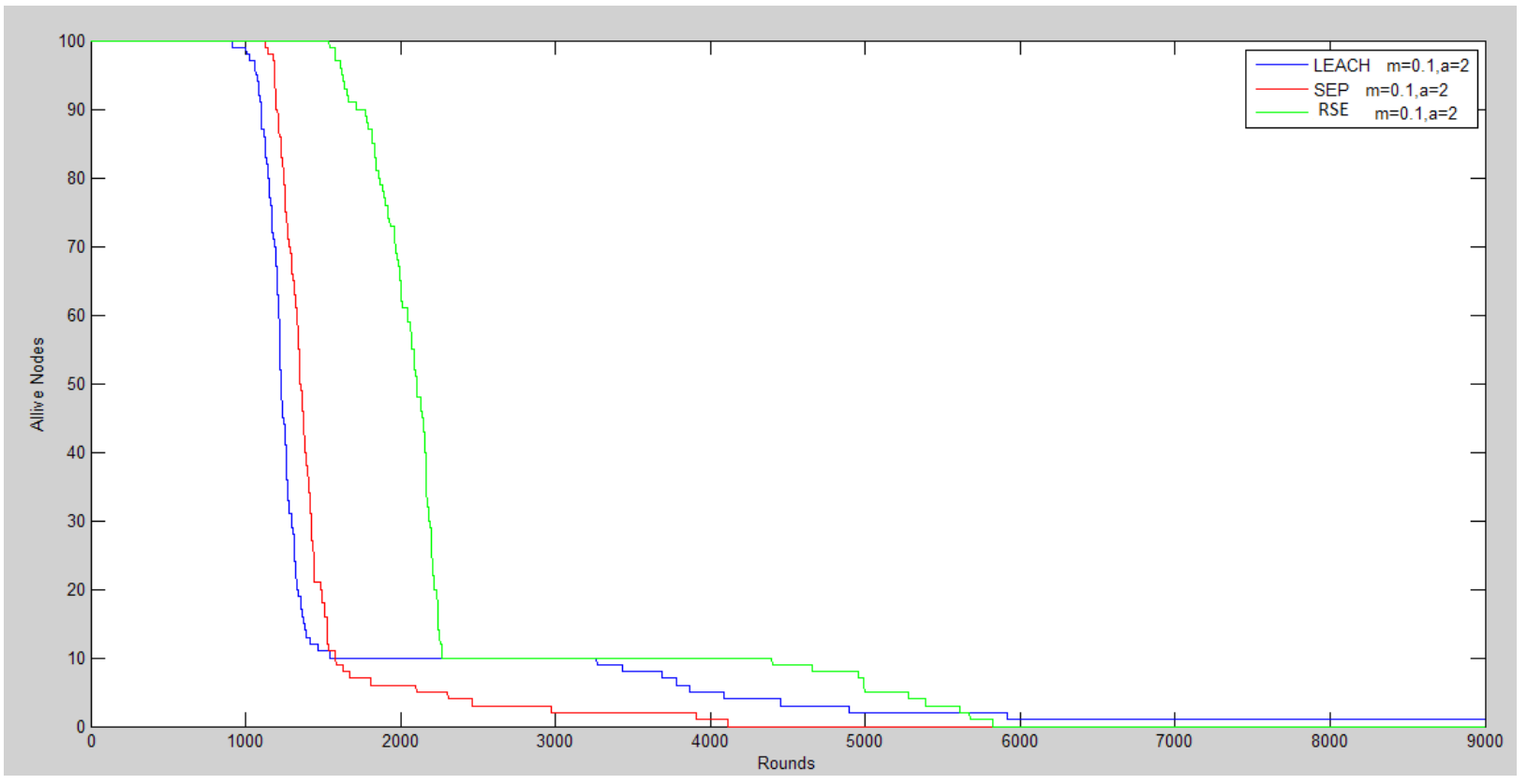

Figure 3. Alive Nodes in LEECH, SEP, RES.

Table 2. Comparison $\mathrm{m}=0$ and $\alpha=2$

\begin{tabular}{llll}
\hline Protocol & $\begin{array}{l}\text { Stability } \\
\text { period }\end{array}$ & $\begin{array}{l}\text { Network } \\
\text { lifetime }\end{array}$ & Throughput \\
\hline Leech & 1018 & 4685 & $2.2 \times 10^{4}$ \\
SEP & 1056 & 3078 & $1.99 \times 10^{4}$ \\
RSE & 1569 & 5099 & $2.9 \times 10^{4}$ \\
\hline
\end{tabular}

Table 3. Comparison between $\mathrm{m}=1$ and $\alpha=2$

\begin{tabular}{llll}
\hline Protocol & $\begin{array}{l}\text { Stability } \\
\text { period }\end{array}$ & $\begin{array}{l}\text { Network } \\
\text { lifetime }\end{array}$ & Throughput \\
\hline Leech & 900 & 5010 & $1.2 \times 10^{4}$ \\
SEP & 1056 & 5022 & $3.99 \times 10^{4}$ \\
RSE & 1178 & 5097 & $4.9 \times 10^{4}$ \\
\hline
\end{tabular}

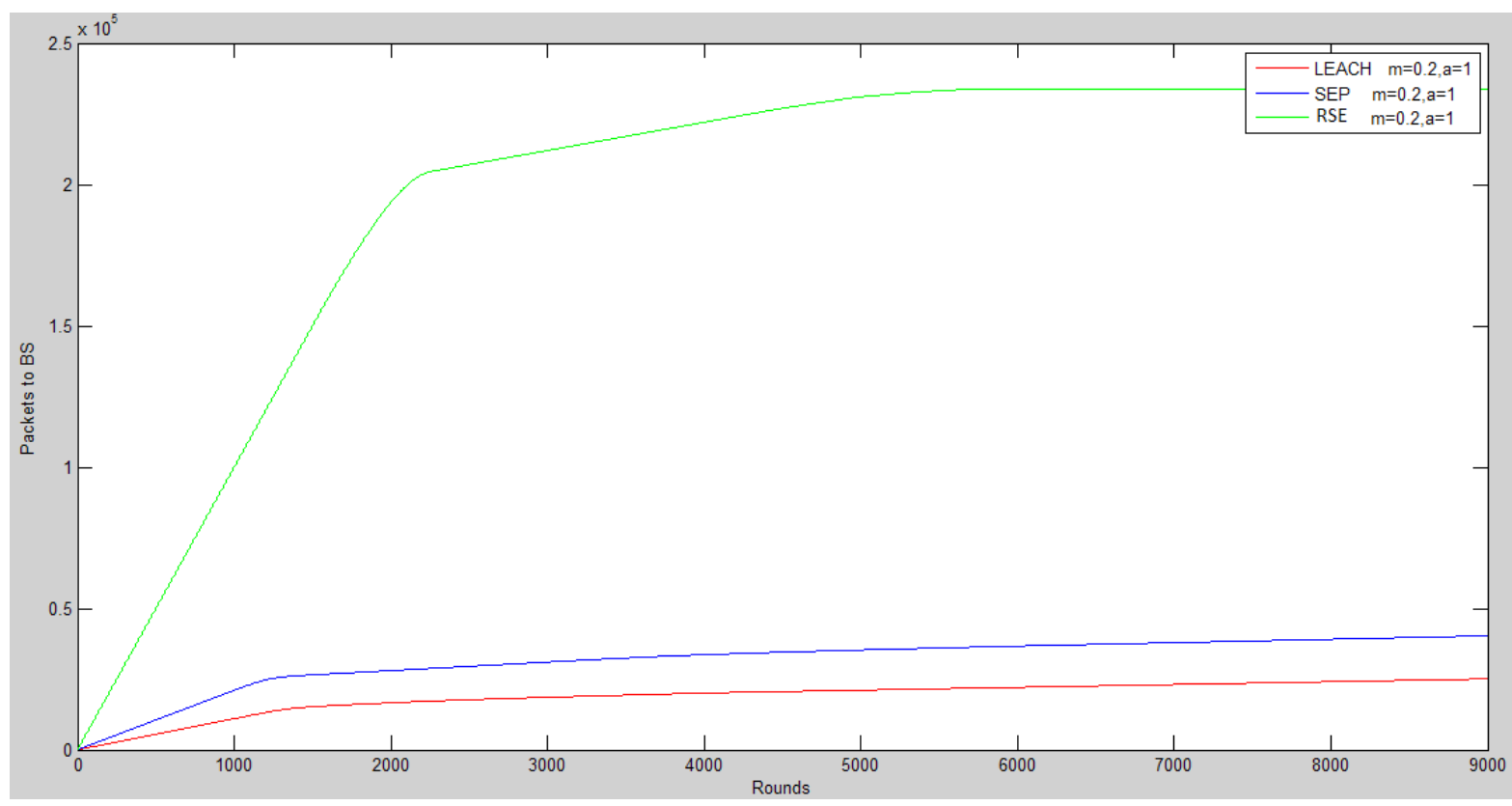

Figure 4. Throughput of LEECH, SEP, RES. 
rounds in simulation. In region specific routing protocol cluster head transform data to the base station. Whereas in other protocol, nodes send data to base station. From the Figure 3, it is well known that region specific routing protocol has better result than existing protocols.

Advanced nodes have more life time than normal nodes. Data aggregation done in cluster head region 1 and region 2 decreases the energy dissipation in the nodes because of clustering techniques. It has more energy ${ }^{12}$ and it dies after long period while compared to normal node. Figure 4, Table 2, Table 3, show the network lifetime and stability period which has increased and it often show better results than LEECH and SEP.

\section{Conclusion}

Region specific election routing protocol is compared with two existing routing protocols which has proven for energy efficiency, throughput, stability period and life time better than two routing protocols. The number of alive nodes after simulation is higher than two existing protocols like SEP and LEECH. The proposed routing protocol is energy efficient while used in real wireless sensor applications.

\section{References}

1. Frost V, Melamed B. Traffic Modeling For Telecommunications Networks. IEEE Communications Magazine (March); 1994. p. 70-81

2. Akyildiz IF, Su W. Sankarasubramaniam Y, Cayirci E. Wireless sensor networks: a survey. Computer Networks. 2002; 38(4):393-422.
3. Younis, Ossama, Fahmy S. HEED: a hybrid, energy-efficient, distributed clustering approach for ad hoc sensor networks. IEEE Transactions on Mobile Computing. 2004 Oct-Dec; 3(4):366-79.

4. Smaragdakis, Georgios, Matta I, Bestavros A. SEP: A stable election protocol for clustered heterogeneous wireless sensor networks. Boston University Computer Science Department; 2004.

5. Loscrì V, Morabito G, Marano S. A Two-Level Hierarchy for Low-Energy Adaptive Clustering Hierarchy (TL-LEACH). Proceedings of IEEE; 2005; 0-7803-9152-7/05.

6. Abbasi AA, Younis M. A survey on clustering algorithms for wireless sensor networks. Computer communications. 2007; 30(14):2826-41.

7. Becchi M. From Poisson Processes to Self-Similarity: an Survey of Network Traffic Models. 2008. Available from: http://www.rajjain.com/cse567-06/ftp/traffic models1.pdf.

8. Javaid N, Yousaf M, Ahmad A, Naveed A, Djouani K. Evaluating impact of mobility on wireless routing protocols. ISWTA2011; 2011. p. 84-9.

9. Wasiq S, Arshad W, Javaid N, Bibi A. Performance evaluation of DSDV, OLSR and DYMO using 802.11 and 802.11p MAC-protocols. INMIC2011; 2011; p. 357-61.

10. Koteeswaran S, Janet J, Kannan E, Visu P. Terrorist. Intrusion Monitoring System using Outlier Analysis Based Search Knight Algorithm. European Journal of Scientific Research. 2012; 74(3):440-9.

11. Rafeh R, Khodadadi M. Detecting Sybil Nodes in Wireless Sensor Networks Using Two-hop Messages. Indian Journal of Science and Technology. 2014; 7(9):1363-72.

12. Ghaffari A. Designing a Wireless Sensor Network for Ocean Status Notification System. Indian Journal of Science and Technology. 2014; 7(6):809-14. 